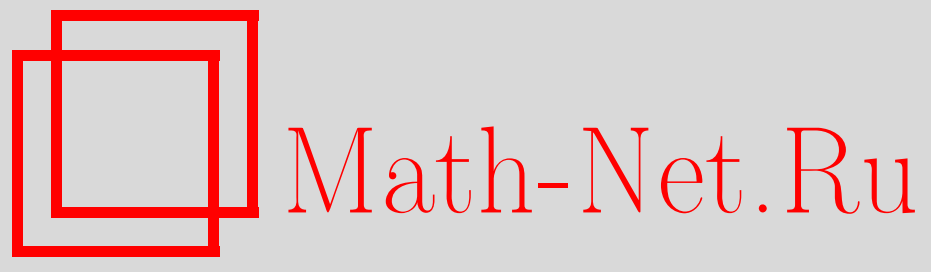

Г. Г. Ильюта, Ряд Ньютона, правило Лейбница и функции конечного порядка, УМН, 2006, том 61, выпуск 4, 189-190

DOI: https://doi.org/10.4213/rm1725

Использование Общероссийского математического портала Math-Net.Ru подразумевает, что вы прочитали и согласны с пользовательским соглашением http://www . mathnet.ru/rus/agreement

Параметры загрузки:

IP: 34.229 .108 .108

26 апреля 2023 г., 12:32:08

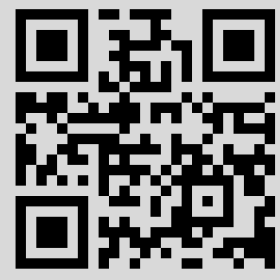




\section{Ряд Ньютона, правило Лейбница и функции конечного порядка}

\section{Г. Г. Ильюта}

Схему использования введенных Ю. И. Маниным и В. В. Шехтманом высших порядков Брюа $B(n, k)$ для кодирования стратов, связанных с интерполяцией конфигурационных пространств [1], можно обобщить следующим образом: для набора элементов $A=\left\{a_{1}, a_{2}, \ldots\right\}$ вещественной алгебры $L$ и линейного функционала $r$ на ней поставим в соответствие каждому $b \in L$ набор знаков $A_{r}(b)=\left(\operatorname{sign} r\left(b a_{1}\right), \operatorname{sign} r\left(b a_{2}\right), \ldots\right)$. Линейные соотношения между $a_{i}$ приводят к соотношениям между $r\left(b a_{i}\right)$, которые налагают условия на $A_{r}(b)$ : если сумма равна 0, то среди ненулевых слагаемых есть положительные и отрицательные. Наборы знаков из $B(n, k)$ получаются для алгебры функций одной переменной, функционала разделенных разностей (р.p.) в $x_{1}, \ldots, x_{n} \in \mathbb{R}$ и $A=\left\{\left(x-x_{i_{1}}\right) \cdots\left(x-x_{i_{n-k+1}}\right): i_{1}, \ldots, i_{n-k+1} \in \bar{n}=\{1, \ldots, n\}\right\}$. Мы изучаем частный случай этой конструкции, связанный с обобщением интерполяционного ряда и р.р. Ньютона

$$
g(Y)=c_{0}+\sum_{i \geqslant 1} c_{i} \prod_{1 \leqslant j \leqslant i} u\left(Y, x_{j}\right), \quad \Delta\left[f(Y) ; X_{n}\right]=\sum_{I} f\left(X_{I}\right) / \prod_{j \in \bar{n} \backslash I} u\left(X_{I}, x_{j}\right),
$$

где $Y=\left\{y_{1}, \ldots, y_{k}\right\}, u(Y, y): S^{k+1} \rightarrow F(S-$ множество, $F-$ поле $), f: S^{k} \rightarrow F, c_{i} \in F$, $X_{n}=\left\{x_{1}, \ldots, x_{n}\right\} \subset F, x_{i}$ различны, $I-k$-подмножество в $\bar{n}, X_{I}=\left\{x_{i_{1}}, \ldots, x_{i_{k}}\right\}$ (случай $u=\operatorname{det}$ рассматривался в [2]). А именно, мы получим обобщенное правило Лейбница для р.р. и приведем простейшие вытекающие из него соотношения для p.p., построенных по функции конечного порядка (аналогия с инвариантами Васильева уточняется ниже). Мы обобщаем р.р. как линейную комбинацию значений функции. Аналогично можно использовать в качестве $r$ обобщения других представлений р.р.: вычет, композиция кратного интеграла и дифференциального оператора, линейно зависящий от $b$ определитель. Также обобщается факт из [1], согласно которому чередуют знак взятые в критических точках р.p. многочлена с вещественными корнями.

ПредЛОЖенИЕ 1 (правило Лейбница). Пустъ $X_{k, n}=X_{n} \cup\left\{x_{1-k}, \ldots, x_{-1}\right\}$ u для $i \leqslant k$ выполнено $\left.u\left(Y, y_{k+1}\right)\right|_{y_{i}=y_{k+1}}=0$. Тогда для любых функиии $f(Y)$ и рлда $g(Y)$ имеем формулу $\Delta\left[g f ; X_{k, n}\right]=\sum_{0 \leqslant i \leqslant n-1} c_{i} \Delta\left[f ; X_{k, n} \backslash\left\{x_{1}, \ldots, x_{i}\right\}\right]$ (из этого равенства при $f=1$ вытекает, что с $c_{i}$ являются линейными комбинациями р.p. ряда $g$ ).

Для $X \subset S, T \subset S^{m+1}$ скажем, что функция $u(Y, y)$ имеет конечный порядок, если для любого $t=\left(t_{1}, \ldots, t_{m+1}\right) \in T$ некоторая нетривиальная линейная комбинация $\sum \lambda_{i}(t) u\left(Y, t_{i}\right)$ равна 0 на всех $k$-подмножествах в $X$, например, если $u$ допускает разделение переменных (р.п.-формулу) вида $u(Y, y)=h_{1}(Y) p_{1}(y)+\cdots+h_{m}(Y) p_{m}(y)$ (характеризацию таких функций можно найти в [3]).

ПредЛожение 2. 1) Если и имеет конечный порядок, $t \in T, t \subset X$, то для любой функиии $f$ имеем соотношение $\sum \lambda_{i}(t) \Delta\left[f ; X \backslash t_{i}\right]=0$.

2) Если и допускает p.n.-формулу, то а) $\operatorname{det}\left(\Delta\left[f ; X \backslash t_{i}\right] \quad p_{1}\left(t_{i}\right) \quad \ldots \quad p_{m}\left(t_{i}\right)\right)=0$, $i=1, \ldots, m+1$, b) при $h_{m}=$ const $, i, j=1, \ldots, m$, имеем соотношение

$$
\Delta[f ; X] \text { const } \operatorname{det}\left(p_{j}\left(t_{i}\right)\right)=\operatorname{det}\left(p_{1}\left(t_{i}\right) \quad \ldots \quad p_{m-1}\left(t_{i}\right) \quad \Delta\left[f ; X \backslash t_{i}\right]\right) .
$$

ПредЛОЖенИЕ 3. Если $F=\mathbb{R}, \operatorname{sign} u\left(x_{i}, x_{j}\right)=-\operatorname{sign} u\left(x_{j}, x_{i}\right)$ для всех $i \neq j$ и чередуются знаки элементов в последовательности $\left\{f\left(x_{i}\right)\right\}$, то для $k \geqslant 1$ чередуются знаки в последовательности $\left\{\Delta\left[f ; x_{i}, \ldots, x_{i+k}\right]\right\}$.

Работа выполнена при поддержке грантов РФФИ 04-01-00762 и НШ-4719.2006.1. 
Оставшаяся часть заметки посвящена примерам. Для достаточно большого $m$ конечность порядка любого многочлена вытекает из определения, в других примерах конечность порядка функции связана с наличием теоремы сложения. Аналогично можно получить соотношения для р.p. с помощью теорем сложения для ортогональных многочленов [4], решений линейных дифференциальных уравнений [5], обобщенных гиперболических функций [6], последовательностей Шеффера [7].

Пусть $S$ - группа, $R$ - ее представление, $p, q \in S, u(p, q)=R\left(p q^{-1}\right)_{i j}$ - матричный элемент. Имеем р.п.-формулу $u(p, q)=\sum R(p)_{i k} R\left(q^{-1}\right)_{k j}$ (аналогично можно использовать представления алгебр Ли). Например, представления

$$
x \mapsto\left(\begin{array}{cc}
1 & x \\
0 & 1
\end{array}\right), \quad\left(\begin{array}{cc}
\cos x & \sin x \\
-\sin x & \cos x
\end{array}\right),\left(\begin{array}{cc}
\operatorname{ch} x & \operatorname{sh} x \\
\operatorname{sh} x & \operatorname{ch} x
\end{array}\right)
$$

приводят к р.p. Ньютона и их тригонометрическим аналогам.

Формула Коши для многочленов Макдональда [8] является р.п.-формулой для результанта двух многочленов одной переменной. Для многомерного результанта (формы Чжоу, записанной в координатах Штифеля) р.п.-формулу можно получить разложением определителя обобщенной матрицы Сильвестра [9] по столбцам, отвечающим одному из многочленов. Подобным образом можно получить р.п.-формулы для результанта из формулы Пуассона [10] и определителей обобщенных матриц Безу и Кронекера [11] (в одномерном случае все эти формулы получаются из формулы Коши различными специализациями многочленов Макдональда). Другими обобщениями одномерного результанта являются двойные многочлены Шуберта и Гротендика. Р.п.-формулами для них являются соответствующие обобщения формулы Коши [12].

Многие теоремы сложения для эллиптических функций Вейерштрасса и Якоби, тэта-функций и их многомерных обобщений можно рассматривать как р.п.-формулы. Например, полагая $u\left(y_{1}, \ldots, y_{k+1}\right)=\sigma\left(\sum y_{i}\right) \prod \sigma\left(y_{j}-y_{k}\right) /\left(\prod \sigma\left(y_{i}\right)\right)^{k+1}$ и используя теорему сложения Фробениуса-Штиккельбергера для $\sigma$-функции Вейерштрасса [13], имеем соотношение для р.p. $\sum_{1 \leqslant i \leqslant k+2}(-1)^{i} u\left(\ldots, x_{i-1}, x_{i+1}, \ldots\right) \Delta\left[f ; X \backslash x_{i}\right]=0$.

Пусть $S$ - группа кос, $v$ - инвариант Васильева порядка $m, u\left(y_{1}, y_{2}\right)=v\left(y_{1} y_{2}\right)$. Выбирая $m+1$ перекрестков в $y_{2}$, видим, что $u$ имеет конечный порядок по отношению к набору $2^{m+1}$ кос, полученных всеми заменами выбранных перекрестков.

Представляет интерес следующий вопрос: существует ли единый подход к упомянутым выше р.п.-формулам, например, можно ли интерпретировать их как теоремы сложения для каких-либо обобщенных групповых структур (такая связь известна, например, для эллиптических функций)?

\section{Список литературы}

[1] Г. Г. Ильюта, Докл. РАН, 347:3 (1996), 306-308. [2] Г. Г. Ильюта, УМH, 58:4 (2003), 149-150. [3] M. Čadek, J. Šimša, Aequationes Math., 40:1 (1990), 8-25. [4] E. Koelink, Special function, q-series and related topics (Toronto, ON, 1995), Fields Inst. Commun., 14, ed. M. Ismail, Amer. Math. Soc., Providence, RI, 1997, 109-129. [5] A. Ungar, Aequationes Math., 26:1 (1983), 104-112. [6] J. Schwaiger, Aequationes Math., 43:2-3 (1992), 198-210. [7] S. Roman, The umbral calculus, Academic Press, New York, 1984. [8] I. Macdonald, Symmetric functions and Hall polynomials, Oxford Univ. Press, New York, 1995. [9] C. D'Andrea, Trans. Amer. Math. Soc., 354:7 (2002), 2595-2629. [10] P. Pedersen, B. Sturmfels, Math. Z., 214:3 (1993), 377-396. [11] B. Mourrain, V. Y. Pan, J. Complexity, 16:1 (2000), 110-180. [12] A. Lascoux, "Notes on interpolation in one and several variables", http://phalanstere.univ-mlv.fr/ ${ }^{\sim a l}$. [13] J. C. Eilbeck, V.Z. Enolskii, E. Previato, Lett. Math. Phys., 63:1 (2003), 5-17.

\section{Г. Г. Ильюта (G. G. Il'yuta)}

Московский государственный открытый педагогический университет им. М. А. Шолохова

E-mail: ilyuta@mccme.ru
Представлено С. М. Гусейн-Заде Принято редколлегией 12.01 .2006 